\title{
Mathematical Model of Pipeline Abandonment and Recovery in Deepwater
}

\author{
Xia-Guang Zeng, ${ }^{1,2}$ Meng-Lan Duan, ${ }^{1,2}$ and Chen $A^{1,2}$ \\ ${ }^{1}$ College of Mechanical and Transportation Engineering, China University of Petroleum, Beijing 102249, China \\ ${ }^{2}$ Offshore Oil and Gas Research Center, China University of Petroleum, Beijing 102249, China \\ Correspondence should be addressed to Xia-Guang Zeng; xiaguangzeng@126.com
}

Received 28 September 2013; Accepted 10 December 2013; Published 29 January 2014

Academic Editor: M. Montaz Ali

Copyright (c) 2014 Xia-Guang Zeng et al. This is an open access article distributed under the Creative Commons Attribution License, which permits unrestricted use, distribution, and reproduction in any medium, provided the original work is properly cited.

\begin{abstract}
In offshore oil and gas engineering the pipeline abandonment and recovery is unavoidable and its mechanical analysis is necessary and important. For this problem a third-order differential equation is used as the governing equation in this paper, rather than the traditional second-order one. The mathematical model of pipeline abandonment and recovery is a moving boundary value problem, which means that it is hard to determine the length of the suspended pipeline segment. A novel technique for the handling of the moving boundary condition is proposed, which can tackle the moving boundary condition without contact analysis. Based on a traditional numerical method, the problem is solved directly by the proposed technique. The results of the presented method are in good agreement with the results of the traditional finite element method coupled with contact analysis. Finally, an approximate formula for quick calculation of the suspended pipeline length is proposed based on Buckingham's Pi-theorem and mathematical fitting.
\end{abstract}

\section{Introduction}

Bad weather is frequent during laying offshore pipelines, so the pipeline abandonment and recovery operation is unavoidable. In offshore oil and gas engineering the pipeline laying engineers need to do detailed mechanical analysis to determine the operation parameters and then make sure that the pipeline will not overstress during the operation. To do the mechanical analysis the mathematical model is a very important problem. In the abandonment operation the $A \& R$ cable lowers a pipeline down to the seabed by a pull head and in the recovery operation lifts it up to the sea level. During the process the pipeline's axial forces, bending moments must be controlled in a reasonable scope to prevent its strength damage. The calculation of these quantities is very useful to guide the operation. So the mathematical model of pipeline abandonment and recovery should be established.

The sketch of the pipeline abandonment and recovery operation is shown in Figure 1. In the processes a pipeline is lifted up from the seabed to the sea surface or put down to the seabed from the sea surface by joint A. The two physical processes are generally called one point lifting and lowering. The processes are mutually inversed and can be described by the same mathematical model. A lot of papers have reported the pipeline installation of mathematical models which are closely related to this operation. Palmer et al. [1] investigated the stresses and configurations of the pipelines being laid from a lay barge over a stinger. They derived equations governing the configuration and solved them by different techniques. Meanwhile they suggested a nondimensionalized governing equation. Mattheij and Rienstra [2] studied the pipeline S-laying model based on a second-order nonlinear differential equation. In the work they explained some difficulties in approximating the numerical solutions. Zhu and Cheung [3] presented an analytical method for finding the elastic deflection of submerged pipelines laid with an adjustable stinger. They claimed that the method costs less computational time than the finite element method (FEM). Guarracino and Mallardo [4] showed a refined analytical analysis of the pipeline S-lay problem. They used a singular perturbation technique and found out a useful analytical solution which took into account the overall effects of 
the pipe cross-section ovalization. Timoshenko et al. [5] provided some analytical and numerical solutions for the pipeline deepwater S-lay which quantified the loading history effects. The analytical solution was fully developed for an arbitrary pipe material model and it was agreed well with the numerical results. Lenci and Callegari [6] developed three simple analytical models for the J-lay problem. By the models the boundary layer phenomenon was correctly detected and the influence of the soil stiffness was studied. By the means of extensive numerical studies, Kashani and Young [7] found that in ultradeepwater pipeline laying problem the installation parameters were sensitive to pipe wall thickness. Gong et al. [8, 9] made a parameter sensitivity analysis of Slay technique for deepwater pipelines. The stiffened catenary theory was applied to establish the governing differential equations. They also presented a numerical iteration method for solving the pipeline configurations, and its validity was further verified by means of a comparison with results obtained from OFFPIPE. Wang et al. [10-12] did some analyses on both S-lay and J-lay problems. They proposed a novel numerical model which could take into account the influence of ocean currents and seabed stiffness. In the model the pipeline was divided into two parts and the continuity of the two parts was guaranteed at the touch down point (TDP). They also presented an analytical model for the pipeline J-lay behavior with plastic seabed. Duan et al. [13] proposed an installation system for deepwater riser S-laying and carried some laboratory scale pipeline lifting experiments by this system. Szczotka [14] studied the pipeline J-lay problem by a modified rigid finite element method (RFEM). A modification of the stiffness coefficients and the corresponding model was proposed. They claimed the model could take into account wave and sea current loads, hydrodynamic forces and material nonlinearity. Yuan et al. [15] presented a novel numerical model for the pipeline Slay problem. They claimed that the model could be used to investigate the overall configuration, internal forces, and strain of the pipelines. On the pipeline abandonment and recovery problem which is very similar to the pipeline laying problem, Andreuzzi and Maier [16] and Datta [17] did the pioneering works. They presented an analytical and a graphical approach for the problem and adopted the finite difference method to analyze the pipeline configurations. Dai et al. [18] studied the configuration of pipelines by the spline collocation method and presented a graphical approach showing the relationship between the configuration and axial forces of the pipeline. Xing et al. [19] continued the research. They built a nonlinear equation system and modeled the pipeline lifting process as a moving boundary problem. By numerical methods the limit moments of some pipelines were obtained. In the researches of pipeline abandonment and recovery, most previous researchers seemed to investigate the problem by a second-order beam equation. However, in our previous research [20] we found that the boundary value problems with the second-order equation cannot tackle the beginning stage of pipeline lifting and the ending stage of pipeline lowing accurately. They produced very different configurations, bending moments at the two stages than the third-order equation boundary value problem

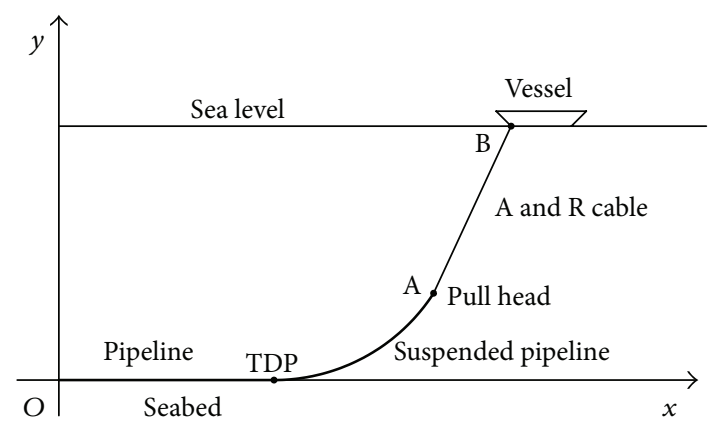

FIgURE 1: Pipeline abandonment and recovery operation.

or Orcaflex. The pipeline in abandonment and recovery operation undergoes a process from a large-angle deflection to a small-angle deflection or from a small-angle deflection to a large-angle deflection. In the problem the boundary condition is moving, which means that it is hard to determine the length of the suspended pipeline segment. The finite element method coupled with contact elements can be used to analyze this problem. However, sometimes it is hard to converge and costs time. Obviously the simple catenary model [21] or stiffened catenary model [22] can never be used to simulate the whole process, so a new mathematical model should be established.

In this paper a mathematical model and a new strategy to tackle the moving boundary without contact analysis are presented. On the other hand the length of the suspended pipeline segment is very important because it can quicken the calculation. So finally a length approximate formula is presented based on Buckingham's Pi-theorem and mathematical fitting.

\section{Mathematical Model}

On the problem the following simplifications are made based on offshore engineering experience $[4,6,17,19]$ : the marine environment is stable, the seabed can be regarded as rigid plane, the lifting and lowering processes are slow, and the material of pipelines is isotropic and always in the elastic state. As shown in Figure 2, the touch down point (TDP, the point where the suspended pipeline contacts with the seabed) is located at the origin $O$ of the Cartesian coordinate system, where $T_{0}$ is the resultant force at joint $\mathrm{A}, H_{0}$ and $V_{0}$ are the horizontal force and the vertical force at the origin, $w$ is the pipeline submerged weight per unit length, $\theta$ is the angle between the pipeline axis and the horizon, and $\theta_{0}$ is the angle between the direction of $T_{0}$ and the horizon. The natural coordinate system is established along the pipeline. It is clear that the physical quantities of the pipeline are the functions of the arc length $s$.

2.1. Governing Differential Equations. The pipeline is regarded as a tensioned beam. There are usually two kinds of differential equations which are used to analyze this problem, a second-order one and a third-order one, and the third-order one is more suitable for the beginning stage of 


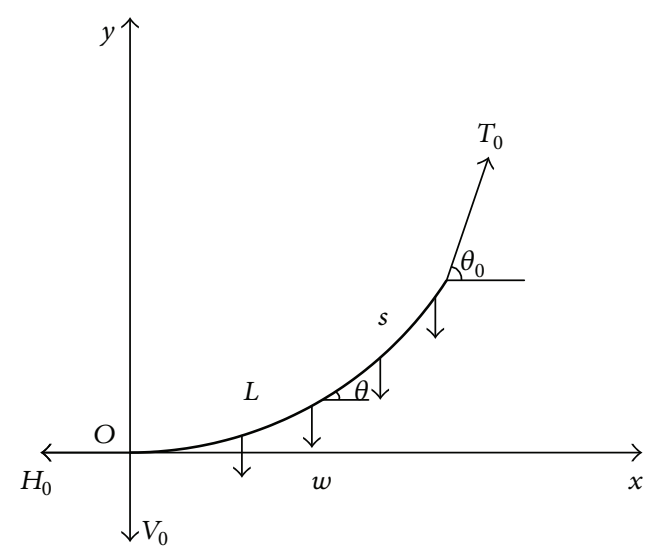

FIGURE 2: Mechanical parameters of the pipeline single point lifting and lowering model.

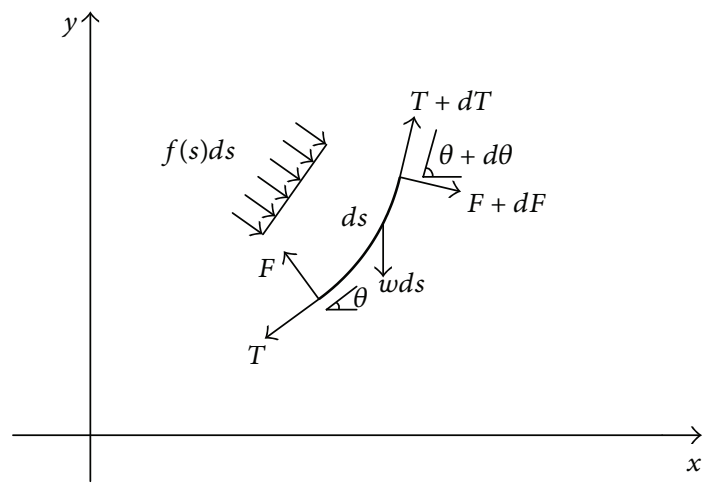

FIGURE 3: Force analysis of a short pipeline segment.

pipeline lifting and the ending stage of pipeline lowing [20]. So in this paper the third-order one has been used. Taking a short segment of the pipeline, as shown in Figure 3, it is easy to deduce the basic governing differential equations [23].

Resolving forces normal to the segment axis leads to

$$
d F-T d \theta+w d s \cos \theta+f(s) d s=0 .
$$

According to $F=d M / d s$, (1) leads to

$$
\frac{d^{2} M}{d s^{2}}-T \frac{d \theta}{d s}+w \cos \theta+f(s)=0 .
$$

According to beam theory, there is the following equation, where $E$ is elastic modulus and $I$ is second moment of area of the pipe cross section:

$$
\frac{M}{E I}=\frac{d \theta}{d s} .
$$

Substituting (3) into (2), then (2) becomes

$$
E I \frac{d^{3} \theta}{d s^{3}}-T \frac{d \theta}{d s}+w \cos \theta+f(s)=0 .
$$

Resolving forces in the segment axis leads to

$$
\frac{d T}{d s}=w \sin \theta
$$

In the pipeline abandonment and recovery problem, $f(s)$ is assumed to be zero, so the governing differential equations for the pipeline lifting and lowering by one point are shown as follows:

$$
\begin{gathered}
E I \frac{d^{3} \theta}{d s^{3}}-T \frac{d \theta}{d s}+w \cos \theta=0, \\
\frac{d T}{d s}-w \sin \theta=0 .
\end{gathered}
$$

2.2. Boundary Conditions. According to the similar problems $[10,24]$, the following boundary conditions are chosen for this problem:

$$
\begin{aligned}
& \text { at the origin: } \theta(0)=0, T(0)=H_{0}=T_{0} \cos \theta_{0}, M(0)= \\
& \text {, } \\
& \text { at the joint: } M(L)=0, T(L)=T_{0},
\end{aligned}
$$

where $M$ is the bending moment of the suspended pipeline segment and $L$ is its length. According to (3), $M(0)=0$ and $M(L)=0$ are equivalent to $(d \theta / d s)(0)=0$ and $(d \theta / d s)(L)=$ 0 .

To sum up, the whole mathematical model for the pipeline abandonment and recovery is the following boundary value problem:

$$
\begin{gathered}
E I \frac{d^{3} \theta}{d s^{3}}-T \frac{d \theta}{d s}+w \cos \theta=0, \\
\frac{d T}{d s}-w \sin \theta=0, \\
\theta(0)=0, \quad \frac{d \theta}{d s}(0)=0, \\
T(0)=H_{0}=T_{0} \cos \theta_{0}, \quad \frac{d \theta}{d s}(L)=0 .
\end{gathered}
$$

\section{Numerical Solutions}

3.1. Numerical Solution Method. It is hard to get the analytical solutions of the mathematical model presented above. So in this research the traditional numerical method, fourth-order accurate finite difference has been used to get the numerical solutions.

3.2. Tackling the Moving Boundary. Notice that the boundary conditions of the model are moving; in another word, the parameter $L$ is usually an unknown before numerical solving. Solving this problem with moving boundary is challenging. The parameter $L$ must be given first then the problem can be solved in numerical methods. The method of variable substitution, $s=\varepsilon L$, has been taken, so the boundary becomes 0 and 1 , and (7) becomes

$$
\begin{gathered}
\frac{E I}{L^{3}} \frac{d^{3} \theta}{d \varepsilon^{3}}-\frac{T}{L} \frac{d \theta}{d \varepsilon}+w \cos \theta=0, \\
\frac{d T}{d \varepsilon}-w L \sin \theta=0,
\end{gathered}
$$




$$
\begin{aligned}
& \theta(0)=0, \quad \frac{d \theta}{d \varepsilon}(0)=0, \\
& T(0)=H_{0}=T_{0} \cos \theta_{0}, \quad \frac{d \theta}{d \varepsilon}(1)=0 .
\end{aligned}
$$

However, the unknown parameter $L$ just goes into the differential equations and still cannot be determined. According to the balance of axial forces at the lifting joint, the equation $T(L)=T_{0} \cos \left(\theta_{0}-\theta(L)\right)$ has been added as a supplementary boundary condition herein. Using this condition $L$ can be calculated in the following steps:

(1) suppose $L=T_{0}\left(\sin \theta_{0} / w\right)$;

(2) solve the boundary value problem (8) by the fourthorder accurate finite difference method or other numerical methods;

(3) get axial force $T_{n}$ at the joint of the pipeline from the results (provided the pipeline divided into $n$ pieces); then compare the value $T_{n}$ and $T_{0} \cos \left(\theta_{0}-\theta_{n}\right)$. If the absolute value of their difference is very small, the $L$ decided in the last step is approximately equal to the length of suspended pipelines and the work is finished. Otherwise, take the following step;

(4) decrease the value of $L$ with a suitable increment if $T_{n}>T_{0} \cos \left(\theta_{0}-\theta_{n}\right)$ and repeat from the second step to the third step until $T_{n}<T_{0} \cos \left(\theta_{0}-\theta_{n}\right)$, or increase the value of $L$ with a suitable increment if $T_{n}<T_{0} \cos \left(\theta_{0}-\right.$ $\left.\theta_{n}\right)$ and repeat from the second step to the third step until $T_{n}>T_{0} \cos \left(\theta_{0}-\theta_{n}\right)$.

Note. the value of the increment controls the precision of the calculation of $L$. To improve the precision, one can repeat the fourth step with a smaller increment. Finally if the increment is smaller than the allowable error, the length parameter $L$ is determined.

\section{Engineering Application}

4.1. Calculation of Pipeline's Physical Quantities. For engineering application, the pipeline's physical quantities during abandonment or recovery, such as pipeline's configuration, bending moments, must be calculated. After numerical calculation of (8), the angle $\theta_{i}$, the tension force $T_{i}$, and the suspended pipeline length $L$ are all known, and then the coordinates of the suspended pipeline can be calculated by the following formulas:

$$
\begin{aligned}
& x_{i}=x_{i-1}+L\left(\varepsilon_{i}-\varepsilon_{i-1}\right) \cos \theta_{i-1}, \\
& y_{i}=y_{i-1}+L\left(\varepsilon_{i}-\varepsilon_{i-1}\right) \sin \theta_{i-1} .
\end{aligned}
$$

And the bending moment of the pipeline can be calculated by the following formula:

$$
M_{i}=\frac{E I}{L} \frac{\theta_{i}-\theta_{i-1}}{\varepsilon_{i}-\varepsilon_{i-1}}
$$

TABLE 1: Basic values of the pipeline in the example.

\begin{tabular}{lcc}
\hline Size $($ inch $)$ & $E I\left(\mathrm{~Pa} \cdot \mathrm{m}^{2}\right)$ & $w(\mathrm{~N} / \mathrm{m})$ \\
\hline 12 & 31399320 & 350 \\
\hline
\end{tabular}

TABLE 2: Different loads on the pipeline in the first case.

\begin{tabular}{lllllllll}
\hline$T_{0}(\mathrm{KN})$ & 100 & 300 & 500 & 700 & 900 & 1100 & 1300 & 1500
\end{tabular}

TABLE 3: Different angles of the loads in the second case.

\begin{tabular}{llllllllllll}
\hline$\theta_{0}\left({ }^{\circ}\right)$ & 70 & 72 & 74 & 76 & 78 & 80 & 82 & 84 & 86 & 88 & 90 \\
\hline
\end{tabular}

4.2. A Numerical Calculation Example. Using MATLAB, (8) with the basic values shown in Table 1 is calculated as an example. More details for the numerical solving method of this problem can refer to solving ODEs with MATLAB [25].

Consider the first case. Suppose that the angle $\theta_{0}$ keeps as a constant of $80^{\circ}$, and the loads are varying in the pipeline abandonment or recovery operation, as shown in Table 2. The results corresponding to these loads are obtained. The configurations of the pipeline and the corresponding bending moments are shown in Figures 4 and 5, respectively. From Figure 4 we know that the mathematical model proposed above can be used in a large scope of water depth (deeper than $3500 \mathrm{~m}$ ) and can simulate the whole lowering and lifting process. And from Figure 5 we know that the bending moment becomes bigger and bigger when the pipeline is lifting. So the most dangerous situation usually happens at the beginning of abandonment or at the end of recovery.

Consider the second case. Keeping the tension $T_{0}$ as a constant of $800 \mathrm{KN}$, varying the angle $\theta_{0}$, as shown in Table 3, the pipeline configurations and bending moments are also obtained by calculations, as shown in Figures 6 and 7 separately.

From Figures 6 and 7 we know that the angle has a great effect on the pipeline configuration and bending moments. It can be seen that while the angle $\theta_{0}$ increases from 70 degree to 90 degree the bending moment of the pipeline increases greatly, especially when $\theta_{0}>84$. And it is clear that the bending moment is more sensitive to the parameter angle $\theta_{0}$ than to the parameter load $T_{0}$.

4.3. Results Comparison. It is necessary to compare the results calculated by the presented model and method with the traditional finite element analysis results. The software called DRICAS is developed by the model and method presented above. Meanwhile Orcaflex is also used, which is a world's leading package for pipeline finite element analysis and it tackles the moving boundary condition by the contact analysis formula [26]. The comparisons of one of their configuration results and bending moments are shown in Figures 8 and 9, respectively. It can be seen that these results are in good agreement. That indicates that the model and the method established for tackling the moving boundary condition in this paper are correct and effective. 


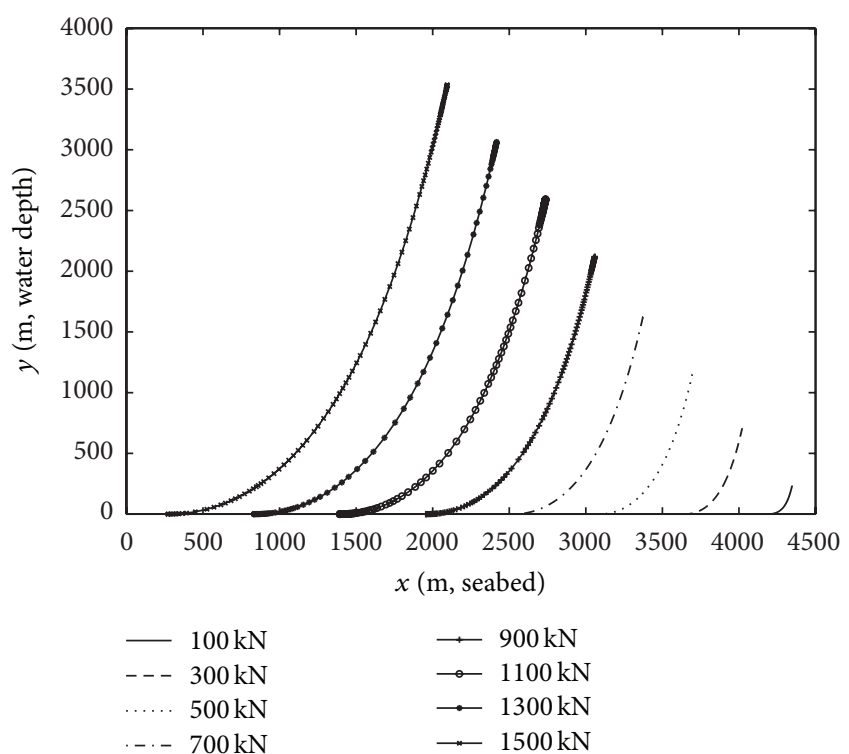

FIGURE 4: Configurations of the suspended pipeline in the first case.

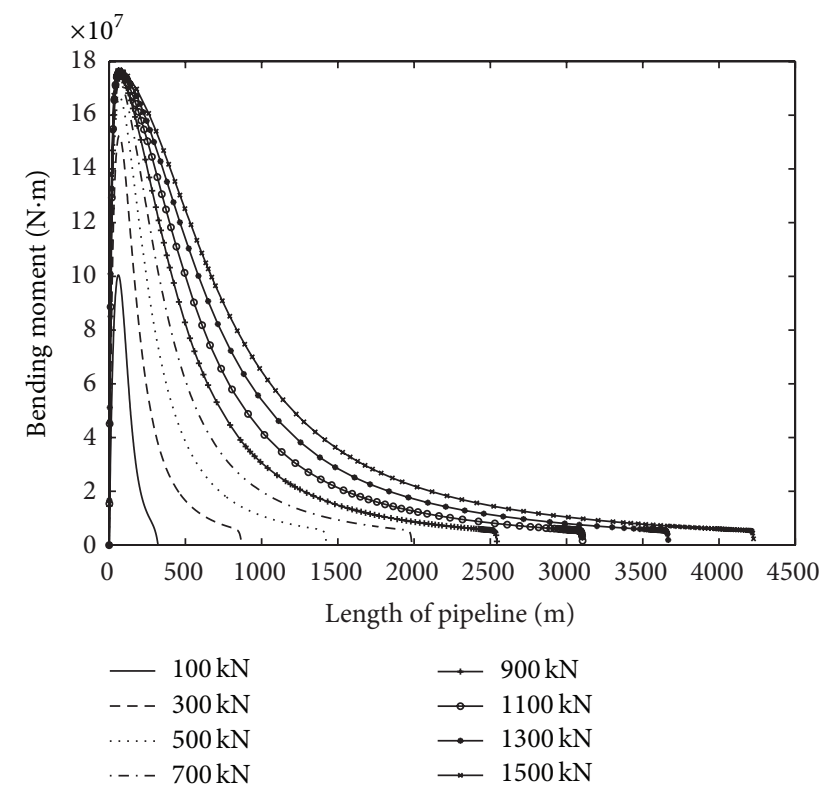

Figure 5: Bending moments of the suspended pipeline in the first case.

\section{Simple Calculation Methods}

5.1. Similarity Criterions of Model Experiment. Sometimes it is necessary to simulate the pipeline recovery and abandonment by model experiments. According to dimensional analysis theory [27], by equation $(d T / d \varepsilon)-w L \sin \theta=0$ and equation $\left(E I / L^{3}\right)\left(d^{3} \theta / d \varepsilon^{3}\right)-(T / L)(d \theta / d \varepsilon)+w \cos \theta=0$, the similarity criterions of such kind of model experiments are obtained; that is, $E I / w L^{3}$ and $T / w L$, respectively. That means if we want to simulate the pipeline abandonment and recovery processes in the laboratory, we should make sure that the values of $E I / w L^{3}$ and $T / w L$ of the model are equal

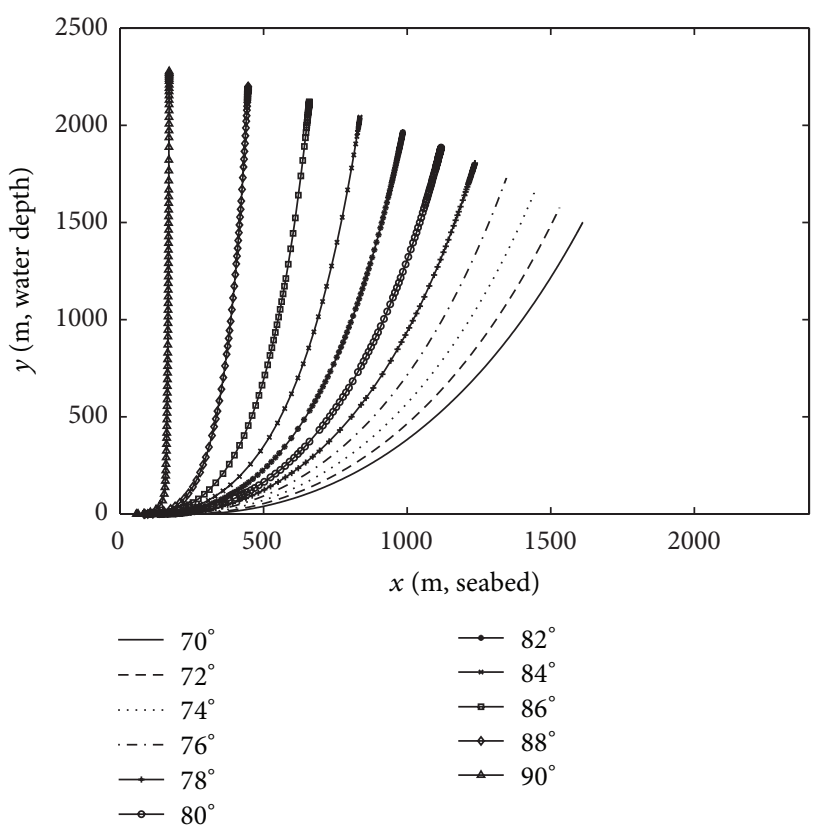

FIgURE 6: Configurations of the suspended pipeline in the second case.

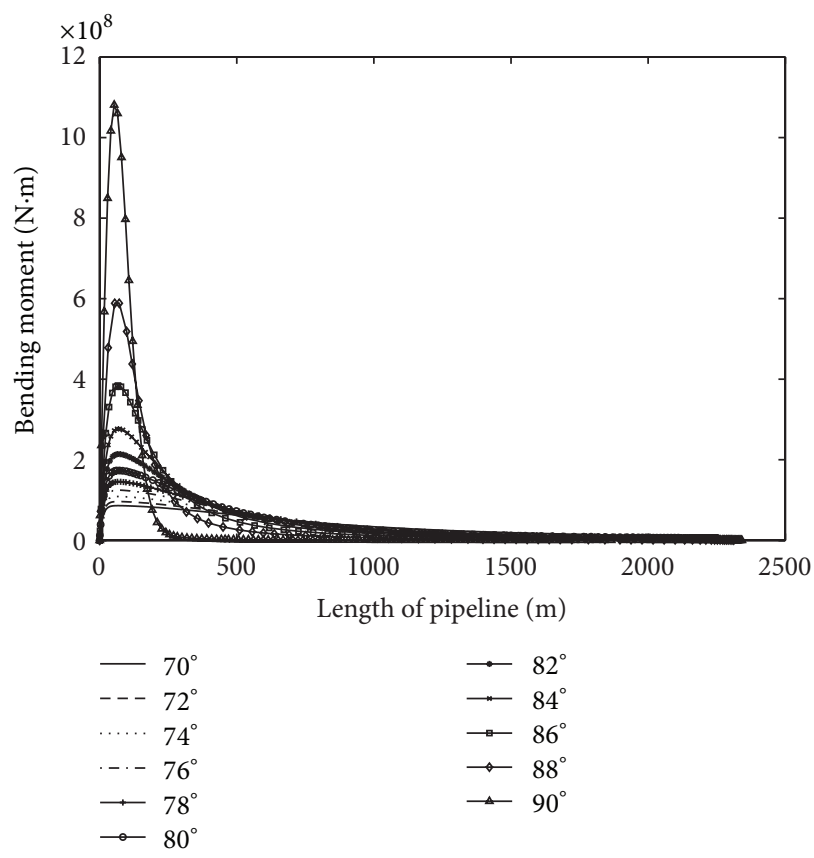

FIGURE 7: Bending moments of the suspended pipelines in the second case.

to the corresponding values of the actual offshore pipeline operation project.

5.2. Approximate Formula of Suspended Pipeline Length. From the numerical calculation procedures it is known that the length of the suspended pipeline is a key parameter of this problem. A simple approximate formula will be very useful to quicken the solving of this boundary value problem. It is 


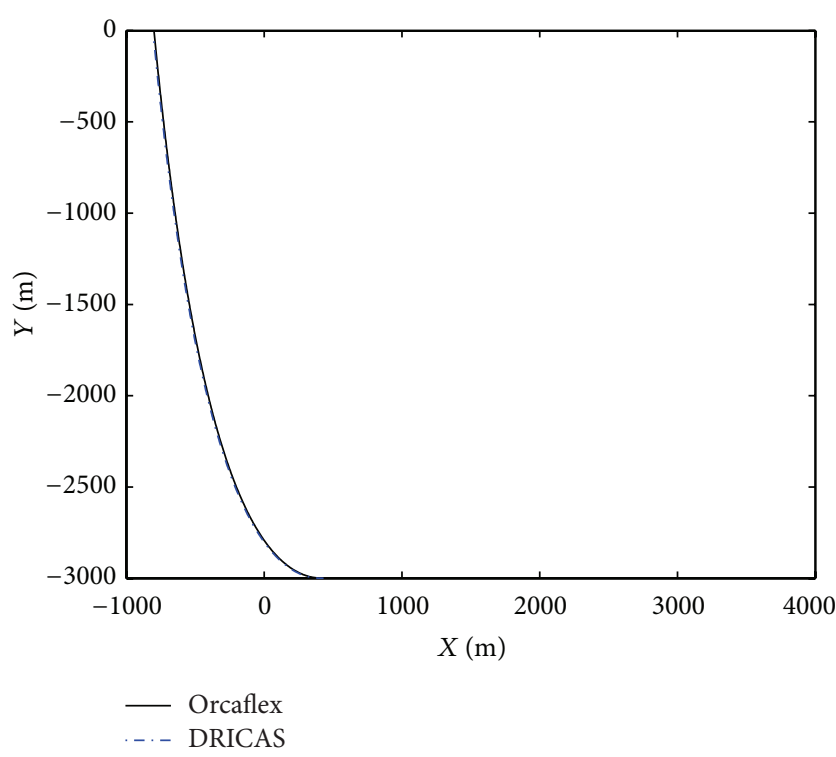

FIGURE 8: Configuration result comparison between DRICAS and Orcaflex.

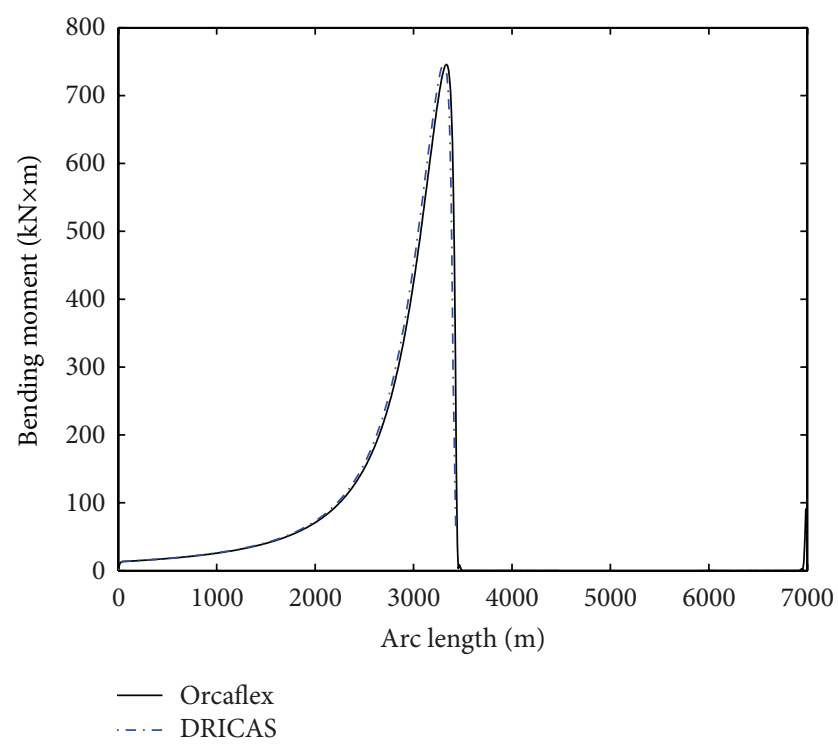

FIGURE 9: Bending moment result comparison between DRICAS and Orcaflex.

known that the length of suspended pipeline is related to $T_{0}$, $E I, w$, and $\theta_{0}$. According to Buckingham's Pi-Theorem [27], a dimensionless function is derived as shown in (11)

$$
\frac{w L}{T_{0}}=f\left(\frac{E I w^{2}}{T_{0}{ }^{3}}, \theta_{0}\right)
$$

To determine (11) completely, the boundary value problem (8) has been solved extensively within the range $0<\pi_{1} \leq$ $500,(76 / 180 \pi)<\pi_{2} \leq(90 / 180 \pi)$, where supposing $\pi_{1}=$ $E I w^{2} / T_{0}{ }^{3}, \pi_{2}=\theta_{0}$, and $\pi_{L}=T / w L$. Using these results the approximate formula for $\pi_{L}$ has been obtained by mathematical fitting as shown in (12). Once $\pi_{L}$ is known, the suspended pipeline length $L$ can be calculated by equation $L=\pi_{L} T_{0} / w$. And if the suspended pipeline length $L$ is known, the steps presented in Section 3.2 can be reduced and hence the computational time for solving the problem:

$$
\begin{aligned}
\pi_{L}= & -0.4085 \pi_{1}^{0.4}+5.162 \pi_{1}^{0.5}-3.895 \pi_{1}^{0.6} \\
- & 0.773 \pi_{2}^{-0.9793}+1.5435, \\
& \left(0<\pi_{1} \leq 1, \frac{76}{180 \pi}<\pi_{2} \leq \frac{90}{180 \pi}\right), \\
= & -0.124 \pi_{1}^{-1.011}-0.773 \pi_{2}^{0.9793}+2.526, \\
& \left(1<\pi_{1} \leq 500, \frac{76}{180 \pi}<\pi_{2} \leq \frac{90}{180 \pi}\right) .
\end{aligned}
$$

\section{Conclusions}

In offshore engineering the pipeline S-laying, J-laying, abandonment and recovery operations can be all governed by (6) which is suitable for the deepwater situation. The differences between these processes are mainly in the boundary conditions.

Reasonable boundary conditions for the problem of pipeline abandonment and recovery are that at the TDP the angle and the bending moment are equal to zero and the tension loading is equal to the loading force horizontal component, and at the joint the bending moment is equal to zero. The whole mathematical model for this problem is (7) or (8), a moving boundary value problem.

The new direct tackling method for the moving boundary of this problem is effective and can get as accurate results as the traditional finite element method coupled with contact analysis.

The similarity criterions for model experiments of pipeline abandonment and recovery are $E I / w L^{3}$ and $T / w L$.

The suspended pipeline length can be calculated first by approximate formula (12) which can quicken the solving of the pipeline abandonment and recovery problem.

\section{Conflict of Interests}

The authors declare that there is no conflict of interests regarding the publication of this paper.

\section{Acknowledgments}

The authors have been supported by the National Basic Research Program of China (no. 2011CB013702) and the National Natural Science Foundation of China (no. 51379214).

\section{References}

[1] A. C. Palmer, G. Hutchinson, and J. W. Ells, "Configuration of submarine pipelines during laying operations," ASME Journal of Engineering for Industry, vol. 96, no. 4, pp. 1112-1118, 1974.

[2] R. M. M. Mattheij and S. W. Rienstra, "On an off-shore pipe laying problem," in Proceedings of the 2nd European Symposium 
on Mathematics in Industry (ESMI '88), H. Neunzert, Ed., pp. 37-55, Springer, 1988.

[3] D. S. Zhu and Y. K. Cheung, "Optimization of buoyancy of an articulated stinger on submerged pipelines laid with a barge," The Ocean Engineering, vol. 24, no. 4, pp. 301-311, 1997.

[4] F. Guarracino and V. Mallardo, "A refined analytical analysis of submerged pipelines in seabed laying," Applied Ocean Research, vol. 21, no. 6, pp. 281-293, 1999.

[5] S. Timoshenko, S. Woinowsky-Krieger, and S. Woinowsky, Theory of Plates and Shells, McGraw-Hill, New York, NY, USA, 1959.

[6] S. Lenci and M. Callegari, "Simple analytical models for the J-lay problem," Acta Mechanica, vol. 178, no. 1-2, pp. 23-39, 2005.

[7] M. Kashani and R. Young, "Installation load consideration in ultra-deepwater pipeline sizing," Journal of Transportation Engineering, vol. 131, no. 8, pp. 632-639, 2005.

[8] S. F. Gong, Y. He, J. Zhou et al., "Parameter sensitivity analysis of S-lay technique for deepwater submarine pipeline," The Ocean Engineering, vol. 4, p. 014, 2009.

[9] S.-F. Gong, K. Chen, Y. Chen, W.-L. Jin, Z.-G. Li, and D.Y. Zhao, "Configuration analysis of deepwater S-lay pipeline," China Ocean Engineering, vol. 25, no. 3, pp. 519-530, 2011.

[10] L.-Z. Wang, F. Yuan, and Z. Guo, "Numerical analysis for pipeline installation by S-lay method," in Proceedings of the 29th ASME International Conference on Ocean, Offshore and Arctic Engineering (OMAE '10), pp. 591-599, ASME, Shanghai, China, June 2010.

[11] L.-Z. Wang, F. Yuan, Z. Guo, and L.-L. Li, "Numerical analysis of pipeline in J-lay problem," Journal of Zhejiang University A, vol. 11, no. 11, pp. 908-920, 2010.

[12] L.-Z. Wang, F. Yuan, Z. Guo, and L.-L. Li, "Analytical prediction of pipeline behaviors in J-lay on plastic seabed," Journal of Waterway, Port, Coastal and Ocean Engineering, vol. 138, no. 2, pp. 77-85, 2012.

[13] M.-L. Duan, Y. Wang, S. Estefen, N. He, L.-N. Li, and B.-M. Chen, "An installation system of deepwater risers by an S-lay vessel," China Ocean Engineering, vol. 25, no. 1, pp. 139-148, 2011.

[14] M. Szczotka, "A modification of the rigid finite element method and its application to the J-lay problem," Acta Mechanica, vol. 220, no. 1-4, pp. 183-198, 2011.

[15] F. Yuan, Z. Guo, L. Li, and L. Wang, "Numerical model for pipeline laying during S-lay," Journal of Offshore Mechanics and Arctic Engineering, vol. 134, no. 2, Article ID 021703, 2011.

[16] F. Andreuzzi and G. Maier, "Simplified analysis and design of abandonment and recovery of offshore pipelines," Ocean Management, vol. 7, no. 1-4, pp. 211-230, 1981.

[17] T. K. Datta, "Abandonment and recovery solution of submarine pipelines," Applied Ocean Research, vol. 4, no. 4, pp. 247-252, 1982.

[18] Y. J. Dai, J. Z. Song, and G. Feng, "A study on abandonment and recovery operation of submarine pipelines," The Ocean Engineering, vol. 18, pp. 75-78, 2000 (Chinese).

[19] J. Z. Xing, C. T. Liu, and X. H. Zeng, "Nonlinear analysis of submarine pipelines during single point lifting," The Ocean Engineering, vol. 20, pp. 29-33, 2002 (Chinese).

[20] X. G. Zeng, M. L. Duan, and J. H. Chen, "Research on several mathematical models of offshore pipe lifting or lowering by one point," The Ocean Engineering, vol. 31, pp. 32-37, 2013 (Chinese).

[21] H. M. Irvine, Cable Structures, MIT Press, Cambridge, Mass, USA, 1981.
[22] D. Dixon and D. Rutledge, "Stiffened catenary calculations in pipeline laying problem," ASME Journal of Engineering for Industry, vol. 90, no. 1, pp. 153-160, 1968.

[23] C. P. Sparks, Fundamentals of Marine Riser Mechanics-Basic Principles and Simplified Analysis, PennWell, 2007.

[24] G. A. Jensen, N. Säfström, T. D. Nguyen, and T. I. Fossen, "A nonlinear PDE formulation for offshore vessel pipeline installation," The Ocean Engineering, vol. 37, no. 4, pp. 365-377, 2010.

[25] L. F. Shampine, I. Gladwell, and S. Thompson, Solving ODEs with MATLAB, 2003.

[26] O. Manual, 2009, http://www.orcina.com/SoftwareProducts/ OrcaFlex/Documentation.

[27] A. A. Sonin, The Physical Basis of Dimensional Analysis, Department of Mechanical Engineering, MIT, Cambridge, Mass, USA, 2001. 


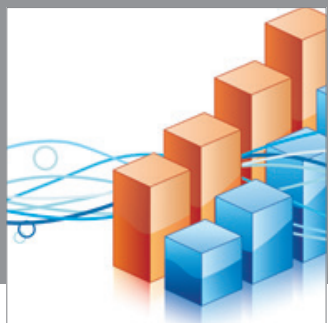

Advances in

Operations Research

mansans

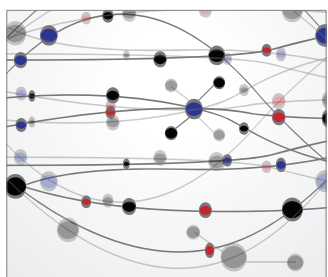

The Scientific World Journal
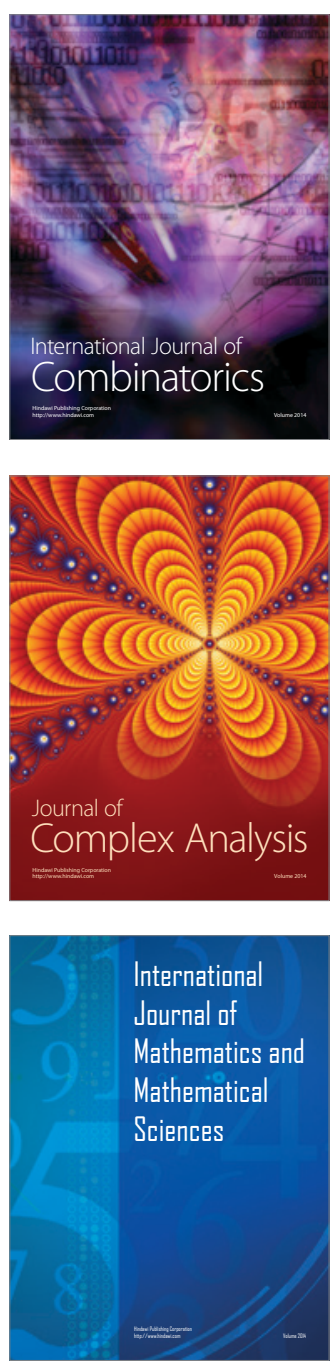
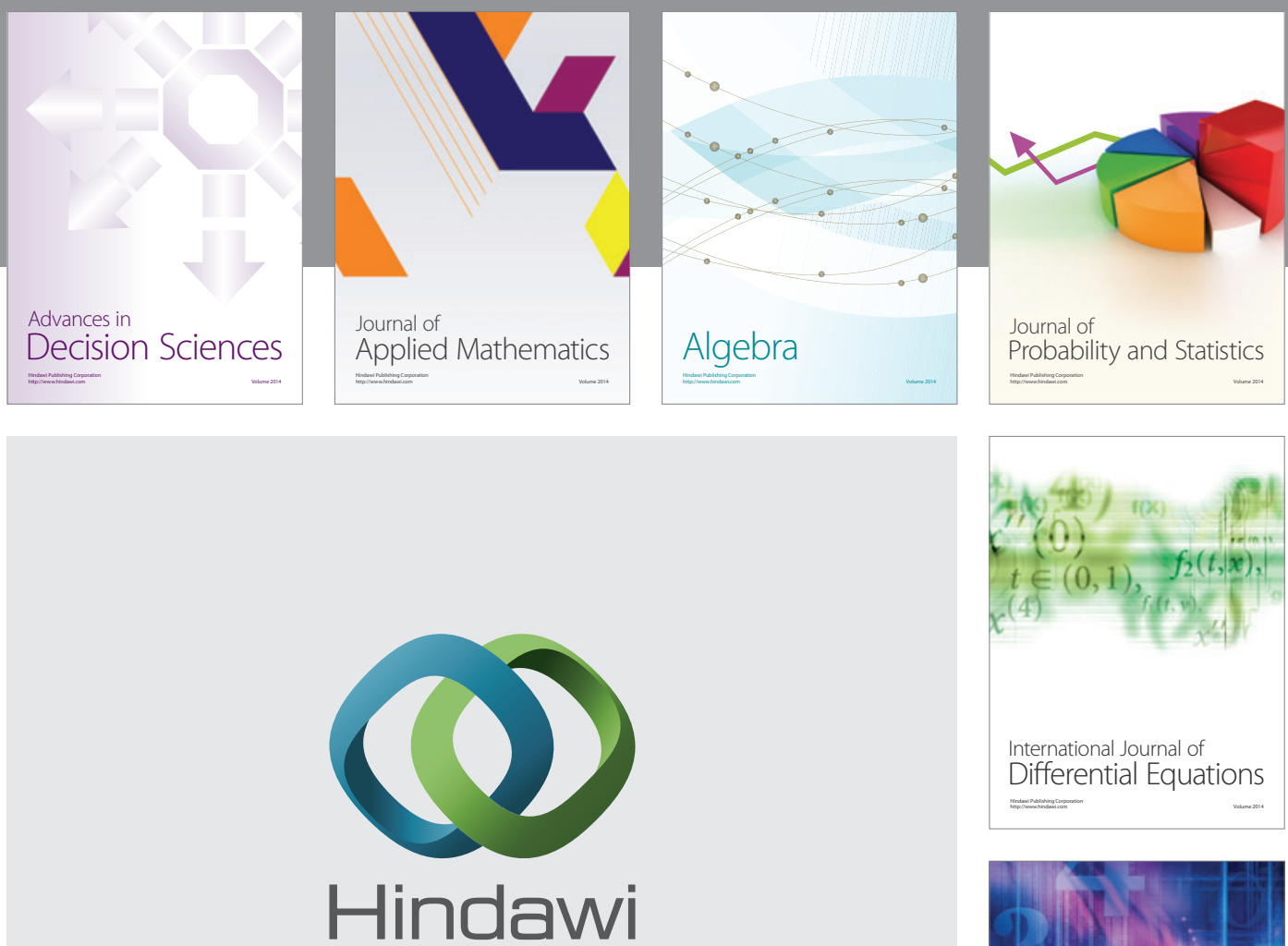

Submit your manuscripts at http://www.hindawi.com
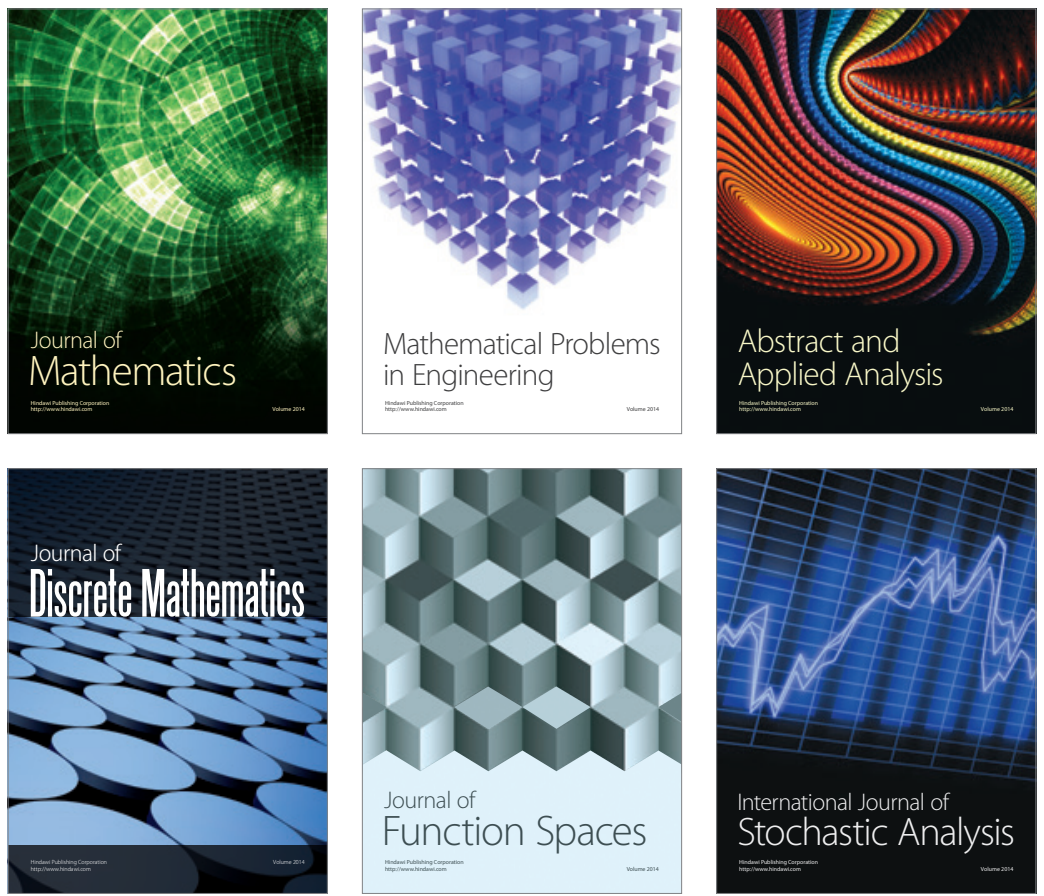

Journal of

Function Spaces

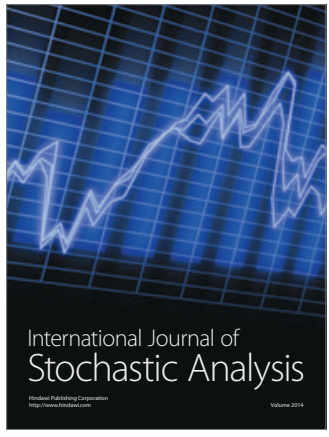

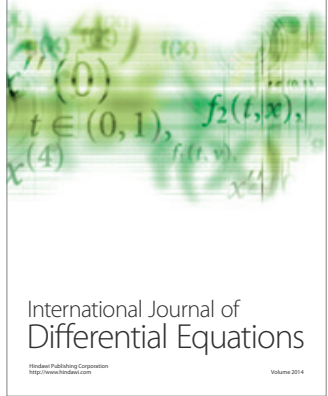
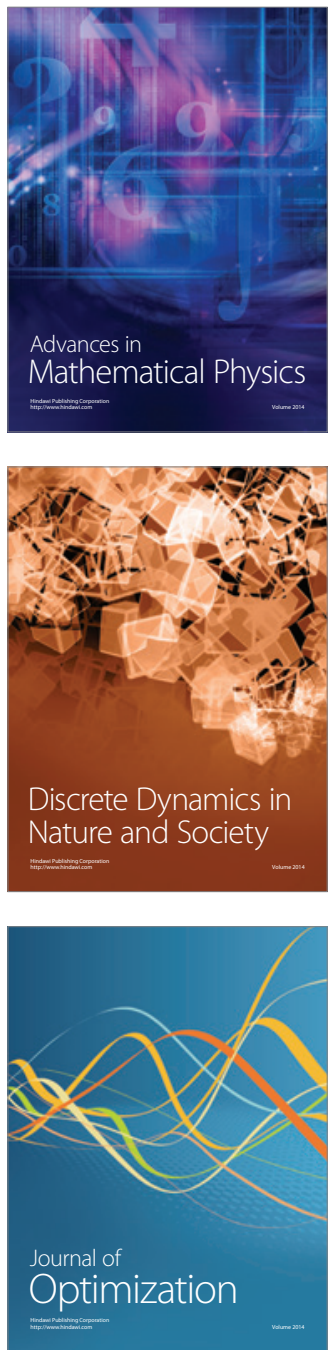